\title{
COMPOSIÇÃO DO COLEGIADO DO CURSO DE PEDAGOGIA EM 1984
}

Presidente-Coordenador: MARCOS EDUARDO KLÜPPEL Vice-Coordenador: MARIA LUCIA FARIA MORO

Departamento de PLANEJAMENTO E ADMINISTRAÇÃO ESCOLAR:

Habilitação: ORIENTAÇÃO EDUCACIONAL:

Titular: MARIA INÊS HAMANN PEIXOTO

Suplente: THERESINHA AZEVEDO CORTÉS

Habilitação: SUPERVISÃO ESCOLAR:

Titular: MARIA ELIZABETH MIGUEL PASSOS

Suplente: JUSSARA MARIA TAVARES PUGLIELLI

Habilitação: ADMINISTRAÇÃO ESCOLAR:

Titular: ODILON CARLOS NUNES

Suplente: DORIS BEATRIZ GONÇALVES PEREIRA

Disciplinas COMUNS DO DEPARTAMENTO:

Titular: NILCÉA MARIA DE SIQUEIRA PEDRA

Suplente: YVELISE FREITAS DE SOUZA ARCO VERDE

Departamento de TEORIA E FUNDAMENTOS DA EDUCAÇÃO

Titular: ALDEMIRO NARDELLI

Suplente: GILDA MOREIRA WEISS 
Departamento de MÉTODOS E TÉCNICAS DA EDUCAÇÃO

Titular: ROSELI CECÍLIA DA ROCHA BAUMEL

Suplente: NELITA FERRAZ DE MELLO SAUNER

Departamento de COMUNICAÇÃO E CIÊNCIAS SOCIAIS

Titular: MILENA MARIA COSTA MARTINEZ

Suplente: GILBERTO AFONSO PAULIN

Departamento de INFORMÁTICA

Titular: DILMA REGINA GRIBOGI KALEGARI

Suplente: ROBERVAL ELOY PEREIRA

Departamento de FILOSOFIA

Titular: JOÃO WANAT

Suplente: INÊS LACERDA ARAÚJO

Departamento de PSICOLOGIA E ANTROPOLOGIA

Titular: JUNGLA MARIA PIMENTEL DANIEL

suplente: THEREZINHA DRUCKER

Departamento de EDUCAÇÃO FísICA

Titular: SONNHILDE ELSE VON DER HEIDE

Suplente: HUGO SALVADOR MIRÓ FERRANTE

Aluna representante do corpo discente:

IVELISE DE PILAR GUIMARÃES.

O Colegiado do Curso de Pedagogia, em várias sessões, contou com a participação e colaboração de professores do Setor de Educação e alunos da graduação e em especial da aluna Márcia Maria Franco Bresolin, Presidente do Diretório Acadêmico Anysio Teixeira. 